\title{
Childhood-onset dense deposit disease: a rare cause of proteinuria
}

\author{
K. Taranta-Janusz $\cdot$ A. Wasilewska $\cdot$ \\ B. Szynaka
}

Received: 4 October 2013/Accepted: 13 November 2013/Published online: 14 December 2013

(C) The Author(s) 2013. This article is published with open access at Springerlink.com

\begin{abstract}
Introduction Dense deposit disease (DDD) is a rare renal disease related to the dysregulation of the alternative pathway of the complement cascade, caused by several factors including the presence of an autoantibody to $\mathrm{C} 3$ nephritic factor, mutations in factor $\mathrm{H}$ and autoantibodies to this protein. DDD is characterized by $\mathrm{C} 3$ accumulation with absent or scanty immunoglobulin deposition.

Case Presentation Herein we report the case of a child with benign course of DDD, who presented with moderate proteinuria and lack of clinical symptoms without immunosuppressive treatment. Laboratory testing revealed moderate proteinuria, normal serum creatinine, total protein, and albumin levels, but significantly decreased serum C3 level. The results of renal biopsy were consistent with DDD. Genetic analysis revealed that the patient carried one copy of the $\mathrm{H} 402$ risk allele of factor $\mathrm{H}$. The level of proteinuria did not change during the follow-up period and no nephrotic syndrome signs occurred. Renal function was stable.

Conclusion In conclusion, a program of urine screening for asymptomatic proteinuria and hematuria to detect children with kidney disease before they experience loss of kidney functions should be considered. Children diagnosed with DDD should have the opportunity to get treatment early on and to be followed very closely.
\end{abstract}

K. Taranta-Janusz · A. Wasilewska $(\square)$

Department of Pediatrics and Nephrology, Medical University of

Białystok, Waszyngtona 17, 15-274 Białystok, Poland

e-mail: annwasil@interia.pl

B. Szynaka

Cathedral of Biostructure Department of Pathomorphology,

Medical University of Białystok, Białystok, Poland
Keywords Dense deposit disease .

Membranoproliferative glomerulonephritis · Child

\section{Introduction}

Dense deposit disease (DDD), also known as membranoproliferative glomerulonephritis type II (MPGN), is a rare disease. It primarily affects children and young adults. According to the new classification of MPGN proposed by Sethi et al. [1], DDD is a negative immunoglobulin and positive $\mathrm{C} 3$ glomerulopathy. It is mostly characterized by MPGN pattern of injury, C3 deposits on immunofluorescence (IF) microscopy and characteristic sausage-shaped, wavy deposits by electron microscopy inside the glomerular basement membrane (GBM) and mesangium [2, 3]. The clinical picture of the disease presents as acute nephritis, proteinuria or nephrotic syndrome. The longterm prognosis to retain native kidney function is usually poor. The pathogenesis of DDD is still obscure, however, it was shown that MPGN resulting from monoclonal gammopathy [4] and dysfunction of the alternative pathway (AP) of complement as the most frequently found factors. Recent discoveries strongly suggest that factor $\mathrm{H}(\mathrm{FH})$ plays a significant role in the pathogenesis of DDD [5, 6].

We report the case of a child with benign course of DDD, who presented with moderate proteinuria and lack of clinical symptoms without immunosuppressive treatment.

\section{Case report}

Our female patient is the third child of non-consanguineous parents. The pregnancy and delivery had been uneventful. The child went on to have normal developmental 
milestones. Her past and family histories were not remarkable for renal diseases.

A previous healthy 8-year-old girl was referred to Department of Pediatrics and Nephrology, Medical University of Bialystok, Poland for proteinuria detected in a screening program at her elementary school. On admission, the girl was in good general condition (body weight $33.6 \mathrm{~kg}$ [75-90 pc], height $133 \mathrm{~cm}$ [75 pc], blood pressure 85/60 mmHg). Physical examination revealed no edema. She was normotensive. Admission laboratory investigations showed leukocyte count $6.7 \times 10^{3} / \mathrm{mm}^{3}$, hemoglobin level $12.7 \mathrm{~g} / \mathrm{dL}$, hematocrit $37.9 \%$, platelet count $257 \times 10^{3} / \mathrm{mm}^{3}$, serum creatinine $0.47 \mathrm{mg} / \mathrm{dL}$, albumin $4.56 \mathrm{~g} / \mathrm{dL}$, cholesterol $260 \mathrm{mg} / \mathrm{dL}, \quad \mathrm{C} 3$ complement $63.1 \mathrm{mg} / \mathrm{dL}$; C4 $20.1 \mathrm{mg} / \mathrm{dL}$, and ASO-tire 112 IU. Antinuclear antibody (ANA), and anti-neutrophil cytoplasmic antibody (ANCA) were negative. Urine protein excretion was $186-680 \mathrm{mg} / 24 \mathrm{~h}$. The sediment contained 3-5 red blood cells per high power field (HPF). Serum immunofixation electrophoresis study for free light chains and urine electrophoresis for monoclonal gammopathy was negative. Glomerular filtration rate (GFR) measured by Schwartz formula was within the normal range $(113 \mathrm{~mL} / \mathrm{min} /$ $1.73 \mathrm{~m}^{2}$ ). Ultrasonography of kidneys showed normalsized kidney with normal corticomedullary distinction.

The patient underwent ultrasound-guided renal biopsy. In the material examined under a light microscope, there were 16 glomeruli with a well-preserved structure. Only in six of them a slight segmental mesangial hypercellularity and an increased matrix mesangium were observed. In these glomeruli some capillaries showed double-contoured basement membrane after Jones' silver stain and PAS stain and a thickening of the walls. No interstitial changes were found. The material for the IF examination contained 13 glomeruli. Moderate or scarce granular deposits of C3 $(+2), \operatorname{IgG}(+2), \operatorname{IgM}(+1)$ (intensity on a scale of $0-4)$ located along the capillary walls of the glomeruli were found. Staining with immunoglobulin $\operatorname{IgA}$ was negative. Light-chain restriction was not documented in the IF microscopy examination. Electron microscopy was performed on six of the glomeruli. Ultrastructural examination revealed scattered amorphous, electron-dense deposits in the GBM under endothelium, in paramesangial and mesangial region, and occasionally with mesangial interposition (Fig. 1a). Fine granular osmiophilic, electron-dense material along the GBM which sometimes formed a homogenous elongated band under endothelium was also observed (Fig. 1b-d). These deposits appeared mainly in the peripherally placed capillaries and occasionally in mesangium matrix.

Genetic analysis was performed in the laboratory of the University of Iowa in the USA. No disease-causing mutations in the coding sequence or splice sites of factor I (FI) and membrane cofactor protein (MCP) were found. However, genetic testing for $\mathrm{FH}$ mutation established that the patient carried one copy of the $\mathrm{H} 402$ risk allele of $\mathrm{FH}$ (Fig. 2). FH-related proteins (CFHR3-CFHR1) testing revealed normal allele. No FI, MCP and FH gene mutations were found in the parents and siblings of the affected child.

During a follow-up period of 3 years, patient's laboratory tests showed urine protein excretion $186-680 \mathrm{mg} /$ $24 \mathrm{~h}$, slight erythrocyturia, decreased serum C3 (62-83.1 $\mathrm{mg} / \mathrm{dL})$, and normal kidney function. The patient was started on an angiotensin-converting enzyme (ACE) inhibitor ( $5 \mathrm{mg} /$ day). Despite moderate proteinuria, she stayed clinically asymptomatic. After 3 years she was lost to follow up for about 1 year. During that time she discontinued medicines but continued to feel well. Laboratory tests revealed normal kidney function and moderate proteinuria.

\section{Discussion}

This is a rare case of a child with DDD and specific polymorphism of the $\mathrm{FH}$ gene, who was diagnosed by a screening program. The girl was in a good general condition at admission and presented with moderate proteinuria and lack of clinical symptoms without immunosuppressive treatment. To date few cases of DDD in children with spontaneous remission have been reported and most of them were treated with steroids [7]. In most of the cases, the disease leads to chronic kidney disease. Lu and colleagues [8] who surveyed 98 DDD patients and their families noted that half of the estimated patients progressed to end-stage renal disease (ESRD) with greater risk for renal failure in females.

Typical features of DDD include the complement profile and the presence of $\mathrm{C} 3$ nephritic factor. The profile usually includes low C3 levels with normal levels of other components. Similar profile of complement was found in our patient. The relationship among C3 and $\mathrm{C} 3 \mathrm{NeF}$ levels and prognosis is still unclear. Nasr et al. [9] have not found any correlation, whereas other authors confirmed that low complement level is a predictive factor of poor prognosis [2].

Understanding of the genetics of DDD is far from complete. The disease is classified as an autoimmune disorder primarily because of the frequent finding of circulating $\mathrm{IgG}$ antibodies known as $\mathrm{C} 3 \mathrm{NeF}$ that is directed against the $\mathrm{C} 3$ convertase, thereby, hindering its degradation. The result of delayed degradation is unchecked activation of the AP, with the consumption of serum $C 3$. Of the genes associated with DDD, the most robust data are available for $\mathrm{FH}$. FH is the primary fluid-phase regulator of the AP of complement. The importance of $\mathrm{FH}$ is reflected 

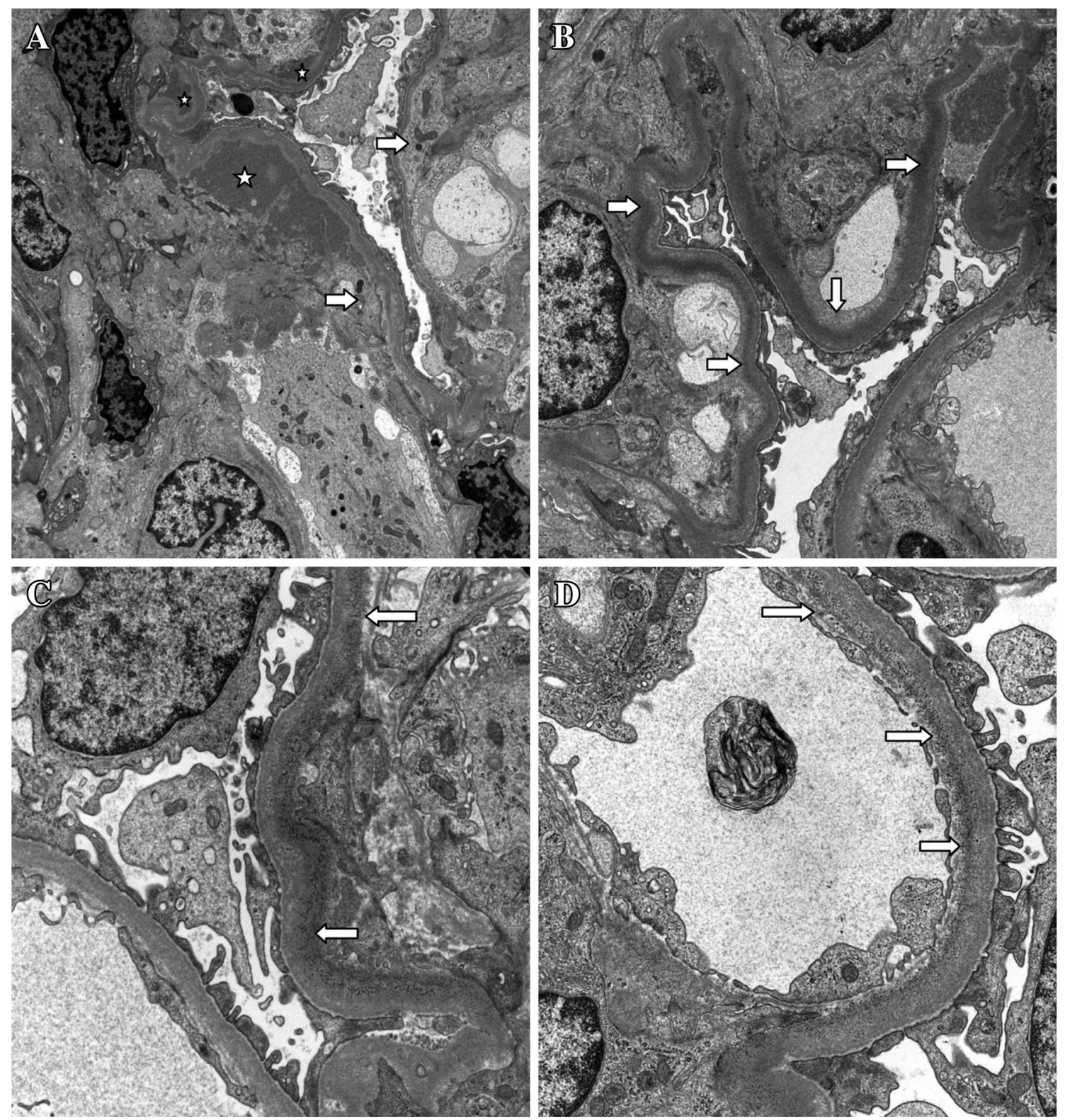

Fig. 1 a Glomerular capillary walls with mesangial interposition (rightwards double arrow) and dense deposits at mesangial and subendothelial location (asterisk). Mag. ×7.000. b Segments of capillary wall with electron-dense material along the glomerular basement membrane which appears as a homogenous band. ME Mag. $\times 7.000$. c The electron-dense deposits of the immune

by the fact that its total absence in mice and pigs leads to unchecked $\mathrm{C} 3$ convertase activity, excessive activation of the alternative complement pathway and eventual development of DDD. Similar outcome is seen in humans. Licht et al. [10] described deletion, $\Delta \mathrm{K} 224$ in two siblings with DDD that resulted in the omission of one amino acid in FH protein and as a consequence, impaired the ability of FH to bind to $\mathrm{C} 3 \mathrm{~b}$ and inactivate the $\mathrm{C} 3$ convertase of the AP. Similarly, an association study showed that $\mathrm{H} 402$ allele of FH was found in $85 \%$ of persons with DDD and much less complexes with fine granules along one of the glomerular basement membrane under endothelium (rightwards double arrow). $\mathrm{ME}$ Mag. $\times 12.000$. d The deposits of fine granular material along the glomerular basement membrane under endothelium (rightwards double arrow). ME Mag. $\times 12.000$

frequently in general population. The H402 allele variant of FH results from a single nucleotide polymorphism which changes the codon encoding the amino acid tyrosine to one encoding histidine. Studies of the H402 allele show that binding of FH to the GBM may be important in protecting it from complement-mediated damage by the AP and that binding of the H402 allele of FH to the GBM is compromised. The study of Leroy et al. [11] performed on eight patients diagnosed with idiopathic MPGN revealed a low C3 level with normal values of FH, FI and surface 


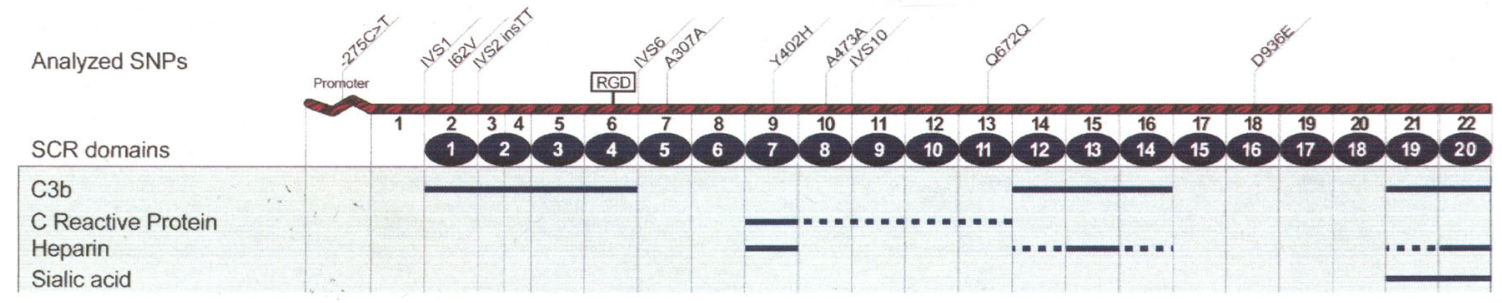

\begin{tabular}{|c|c|c|c|c|c|c|c|c|}
\hline $\begin{array}{c}\text { Single } \\
\text { Nucleotide }\end{array}$ & $\begin{array}{l}\text { Amino } \\
\text { Acid }\end{array}$ & \multicolumn{3}{|c|}{$\begin{array}{c}\text { MPGN2 } \\
\text { Common Patient Haplotype }\end{array}$} & \multicolumn{4}{|c|}{ Patient MPGN2-102 } \\
\hline & & Nucleotide & Amino Acid & P Value $^{\star}$ & Allele -1 & Amino acid & Allele 2 & Amino acid \\
\hline rs800292 & $162 \mathrm{~V}$ & G & V62 & 0.0037 & G & V62 & G & 162 \\
\hline IVS -18ins TT & Intronic & - & - & 0.0014 & - & & - & \\
\hline rs1061170 & $\mathrm{Y} 402 \mathrm{H}$ & C & $\mathrm{H} 402$ & 0.00043 & C & $\mathrm{H} 402$ & $\mathrm{~T}$ & Y402 \\
\hline rs2274700 & A473A & G & A & 0.000022 & G & A & A & A \\
\hline
\end{tabular}

* Based on comparison to 131 controls without age-related macular degeneration

Fig. 2 Factor $\mathrm{H}$ mutation screening of our patient

expression of MCP with genetically confirmed heterozygous mutation of $\mathrm{FH}$, similar to the results of our patient. Boon and co-workers [12] described in their study that individuals who carry FH mutation on one allele in combination with the presence of the $\mathrm{H} 402$ on the other allele developed age-related macular degeneration (AMD). Servais et al. [13] found that genetic background of the patients may also influence the disease manifestation since common genetic variants including single nucleotide polymorphisms in the FH gene are associated with DDD.

In contraposition of MPGN I, there was no constant glomerular finding by light microscopy with a variation of microscopic pattern. Only 6 of 16 of the glomeruli presented focal segmental mesangial proliferation and occasional thickening of the GBM with the double-contoured basement membrane. So, picture in light microscope supports DDD. However, IF study was rather typical for MPGN I with deposits of $\mathrm{C} 3$ complements $(+2), \operatorname{IgG}(+2)$ and $\operatorname{IgM}(+1)$, instead of typical for DDD striking C3 positivity along the capillary loops.

The hallmark of ultrastructural examination that supports diagnosis of immune complexes disease (MPGN) is the presence of mesangial, paramesangial and subendothelial electron-dense deposits as well as fine granular material along the GBM under endothelium. These linear deposits might suggest light-chain deposition disease (LCDD), but do not correspond with IF. These deposits are also seen in early stages of DDD in children. Deposits in DDD usually present more as a homogenous than granular, and showed ribbon-like pattern within the GBM. Localization of deposits in peripheral capillary loops with results of IF study and negative light-chain restriction predispose to DDD diagnosis.
Sethi et al. [1] found that $41 \%$ of patients with DDD had serum and/or urine electrophoresis studies positive for monoclonal gammopathy. The authors suggested that DDD may often be the first sign of the underlying lymphoplasmacytic disorder. It was found that DDD might be the complication of monoclonal gammopathy of undetermined significance. In this base the monoclonal Ig acts as an autoantibody to $\mathrm{FH}$ or other complement regulating proteins that on a permission genetic background (the $\mathrm{H} 402$ allele of FH) leads to dysregulation of the AP with subsequent MPGN. We looked for monoclonal gammopathy in our patient, however, the electrophoresis was negative and no clinical signs were observed. We wish we used serum immunofixation electrophoresis method which is more sensitive.

The prognosis of patients with DDD is generally quite poor, especially in patients with nephritic syndrome, who do not respond to treatment. Although there are some reports of spontaneous remissions of DDD, this is still uncommon, as most of the patients progress to ESRD [14]. Specific mutations and variants in the FH gene are associated with broad range of phenotypes, from early onset renal diseases with high mortality rates to disorders limited to the eye [15]. Differences in clinical phenotypes between our patient and others might depend on variants in the $\mathrm{FH}$ gene. The H402 allele of FH is very frequent in the population. There is a higher frequency in AMD and also in C3 glomerulopathy, but this variant alone is not responsible for the complement overactivation in DDD. Severity of clinical features may depend partly on specific FH sites affected by mutation. We suppose that another reason for a stable clinical course might be the absence of detectable abnormalities of other factors influencing systemic alternative complement pathway activation, i.e., C3Nef, FI. However, 
direct influence of this gene abnormality upon our patient's renal condition remains unclear. Further studies are necessary to find out whether a particular clinical phenotype or histopathology pattern predicts response to therapy who should be treated and who will have a relatively benign disease course.

In conclusion, a program of urine screening for asymptomatic proteinuria and hematuria to detect children with kidney disease before they experience loss of kidney functions should be considered. Children diagnosed with DDD should have the opportunity to get treatment early on and be followed very closely.

Acknowledgments We wish to thank Prof. Richard Smith (University of Iowa Hospitals and Clinics) for performing the FI, MCP and factor $\mathrm{H}$ gene mutation screening in our patient. None of the authors have any financial or personal relationships with other people or organizations that could inappropriately influence this work. Written informed consent was obtained from the patient for publication of this case report and any accompanying images. A copy of the written consent is available for review by the Editor-in-Chief of this journal.

\section{Conflict of interest None.}

Open Access This article is distributed under the terms of the Creative Commons Attribution License which permits any use, distribution, and reproduction in any medium, provided the original author(s) and the source are credited.

\section{References}

1. Sethi S, Fervenza FC (2011) Membranoproliferative glomerulonephritis: pathogenetic heterogeneity and proposal for a new classification. Semin Nephrol 31(4):341-348

2. Appel GB, Cook HT, Hageman G et al (2005) Membranoproliferative glomerulonephritis type II (dense deposit disease): an update. J Am Soc Nephrol 16(5):1392-1403
3. Smith RJ, Alexander J, Barlow PN et al (2007) New approaches to the treatment of dense deposit disease. J Am Soc Nephrol 18(9):2447-2456

4. Sethi S, Sukov WR, Zhang Y et al (2010) Dense deposit disease associated with monoclonal gammopathy of undetermined significance. Am J Kidney Dis 56(5):977-982

5. Sugimoto K, Fujita S, Miyazaki K, Okada M, Takemura T (2012) C3 glomerulonephritis associated with a missense mutation in the factor H gene. Tohoku J Exp Med 227(3):211-215

6. Tortajada A, Yébenes H, Abarrategui-Garrido C et al (2013) C3 glomerulopathy-associated CFHR1 mutation alters FHR oligomerization and complement regulation. J Clin Invest 123(6): 2434-2446

7. Kim MS, Hwang PH, Kang MJ, Lee DY (2010) A case of regression of atypical dense deposit disease without $\mathrm{C} 3$ deposition in a child. Korean J Pediatr 53(7):766-769

8. Lu DF, Moon M, Lanning LD, McCarthy AM, Smith RJ (2012) Clinical features and outcomes of 98 children and adults with dense deposit disease. Pediatr Nephrol 27(5):773-781

9. Nasr SH, Valeri AM, Appel GB et al (2009) Dense deposit disease: clinicopathologic study of 32 pediatric and adult patients. Clin J Am Soc Nephrol 4(1):22-32

10. Licht C, Heinen S, Józsi M et al (2006) Deletion of Lys224 in regulatory domain 4 of factor $\mathrm{H}$ reveals a novel pathomechanism for dense deposit disease (MPGN II). Kidney Int 70(1):42-50

11. Leroy V, Fremeaux-Bacchi V, Peuchmaur M et al (2011) Membranoproliferative glomerulonephritis with $\mathrm{C} 3 \mathrm{NeF}$ and genetic complement dysregulation. Pediatr Nephrol 26(3): 419-424

12. Boon CJ, Klevering BJ, Hoyng CB et al (2008) Basal laminar drusen caused by compound heterozygous variants in the $\mathrm{CFH}$ gene. Am J Hum Genet 82(2):516-523

13. Servais A, Noël LH, Frémeaux-Bacchi V, Lesavre P (2013) C3 glomerulopathy. Contrib Nephrol 181:185-193

14. Ikeda M, Honda M, Hasegawa O (2001) Another example of spontaneous improvement in a case of dense deposit disease. Pediatr Nephrol 16(7):609-610

15. Boon CJ, van de Kar NC, Klevering BJ et al (2009) The spectrum of phenotypes caused by variants in the $\mathrm{CFH}$ gene. Mol Immunol 46(8-9):1573-1594 